\title{
Knowledge and Practice of Nursing Personnel on COVID-19; ONLINE DESCRIPTIVE STUDY
}

\author{
Muralidharan S. ${ }^{1}$ \\ Associate Professor \\ Dept. of Medical Surgical Nursing \\ Maharaja Agrasen Nursing College \\ Bahadurgarh, Haryana \\ Priya Sharma ${ }^{2}$
}

\author{
Assistant Professor \\ Dept. of Community Health \\ Nursing \\ Shri Vinoba Bhave College of \\ Nursing, Silvassa, D\&NH \\ Sujeeta Malik ${ }^{3}$
}

\author{
Nursing Tutor \\ Dept. of Community Health \\ Nursing \\ Maharaja Agrasen nursing College \\ Bahadurgarh, Haryana
}

\begin{abstract}
Background \& objective: The global pandemic always has a huge impact on most countries, but in India, it seems to be worse than most - the crisis is overburdening India's health care system. COVID-19 is an emerging public health problem which threaten the life of over 2.4 million people in World. ${ }^{[1]}$ The present study aimed to assess knowledge and practice of nursing personnel on COVID -19. Methodology: The descriptive research design was used for this study. Data was collected by using WhatsApp messenger and Facebook messenger among nursing personnel who belong to 18 years of age and above. Result: Of 216 nursing personnel approached, 200 responded. The study results revealed that majority $(\mathbf{4 4 . 5 \% )}$ ) of nursing personnel had average knowledge and $43.5 \%$ had good knowledge on COVID-19. Majority $96.5 \%$ of nursing personnel had excellent practice toward COVID-19. There was weak correlation between knowledge and practice on COVID-19 as measured by Spearman Rank Correlation test $(r=0.2817)$. Conclusion \& Interpretation: The study concluded that nursing education is suggested to advance knowledge of nursing personnel hence practice can be improved.
\end{abstract}

Keywords:- Knowledge, Practice, Nursing Personnel, COVID-19

\section{INTRODUCTION}

Already in the first two decades of the 21st century, the world has been sharply reminded time after time of the degree to which people in all countries and on all continents remain chronically vulnerable to infectious diseases, known and unknown. ${ }^{[1]}$ In 2009, a novel influenza virus (H1N1), created the first influenza pandemic of the 21 st century. In 2012-2013, a new virus surfaced in the Middle East caused an epidemic of Middle East respiratory syndrome which spread fatally into many countries beyond that region. [2] About 8 years after the MERS-CoV epidemic, the current outbreak of COVID-19 in Wuhan City, Hubei Province, China, has emerged as a global outbreak and significant public health issue. Coronavirus infection is an ongoing pandemic which is caused by severe acute respiratory syndrome coronavirus 2 (SARS-CoV2). ${ }^{[3]}$ On January 30, 2020, World Health Organization stated COVID-19 as a public health emergency of international concern and was identified as a pandemic on 11 March 2020. It affected 5,891,182 people in world. On
25 March, more than 423,000 cases of COVID-19 have been reported in more than 190 countries and territories, resulting in more than 18,900 deaths and more than 109,000 recoveries. In India 182143 cases are reported of COVID-19 on 31May, 2020 and 5164 death have been reported. ${ }^{[4]}$ Health care workers play a critical role, not only in the clinical management of patients, but also in ensuring adequate infection prevention and control measures are implemented in healthcare facilities. Initial surveillance focuses primarily on patients with severe disease, and as such, the full spectrum of diseases, including the extent and fraction of mild or asymptomatic infection that do not require medical attention and the role they may play in secondary transmission are not clear. Nurses are the front line in contact with patients and are an important source of exposure to infected cases in health care settings; thus, nurses are expected to be at high risk of infection. ${ }^{[5]}$ According to Centres for Disease Control and Prevention, more than 62,000 doctors, nurses and other health care providers on the front lines of the U.S.'s COVID-19 crisis have been infected, and at least 291 have died. ${ }^{[6]}$ By the end of January, the WHO and Centers for Disease Control and Prevention (CDC) had published recommendations for the prevention and control of COVID-19 for nursing professionals. ${ }^{[7]}$ At least 156 nurses were found positive for COVID-19 on 22 April,2020 in India. ${ }^{[8]}$

Understanding 2019-nCoV infection among healthcare workers and the risk factors for adverse outcomes is important not only for characterizing virus transmission patterns and risk factors for infection, but also for preventing future infection of healthcare workers and other patients, for informing and updating infection prevention and control measures at healthcare facility and national level and for reducing secondary 2019-nCoV transmission within healthcare settings. ${ }^{[6]}$ A cross-sectional study was conducted to assess the knowledge and perception among Health care worker about COVID-19. The data was collected by using 23-item survey instrument through social media. The result revealed that among 453 HCWs; $51.6 \%(n=234)$ were male, $32.1 \%(n=147)$ were aged 25-34 years, and most were doctors $(n=137,30.2 \%)$ and medical students $(\mathrm{n}=134,29.6 \%)$. Most participants $(n=276,61.0 \%)$ used social media to obtain information on COVID-19. Majority $(n=276,61.0 \%)$ of HCWs had poor knowledge of its transmission and symptom onset $(n=288$, $63.6 \%$ ) and showed positive perceptions of COVID-19. 
Thus the study concluded as the global threat of COVID-19 continues to emerge, it is critical to improve the knowledge and perceptions of $\mathrm{HCWs}^{[8]}$. Ronald Olum et. al. conducted an online cross sectional study to investigate knowledge and practice among 581 health care workers through WhatsApp Messenger in Uganda among HCWs aged 18 years. The study result showed that among $581 \mathrm{HCWs}$ approached, $136(23 \%)$ responded. Majority (62\%) were medical doctors and $(92 \%)$ had at least a bachelor's degree. Overall 69\% $(\mathrm{n}=94)$ had sufficient knowledge, $21 \%(\mathrm{n}=$ 29) had positive attitude, and $74 \%(\mathrm{n}=101)$ had good practices toward COVID-19. The study concluded that continued professional education is needed among HCWs to improve knowledge of HCWs hence averting negative attitudes and promoting positive preventive and therapeutic practices. [9] Nurses provide education and support that enables patients to manage their care. The present research study aimed to assess the knowledge and practice of nursing personnel on COVID - 19.

\section{Objectives of the Study:}

- To assess the knowledge of nursing personnel regarding COVID 19

- To assess the practice of nursing personnel regarding COVID 19

- To co-relate knowledge and practice of nursing personnel regarding COVID 19

- To find out the association of knowledge scores of nursing personnel with their baseline characteristics.

- To find out the association of practice scores of nursing personnel with their baseline characteristics.

\section{Hypotheses}

- $\mathbf{H}_{1}$ : There is a significant positive correlation between knowledge score and Practice score of nursing personnel regarding COVID 19 at $r>0$.

- $\mathbf{H}_{2}$ : There is a significant association of knowledge scores of nursing personnel with their selected demographic variables.

- H3: There is a significant association of practice scores of nursing personnel with their selected demographic variables.

\section{METHODOLOGY}

RESEARCH APPROACH: In this study, quantitative research approach was used to assess the knowledge and practice regarding COVID 19.

RESEARCH DESIGN: A cross-sectional descriptive research design was adopted for this study to assess the knowledge and practice of nursing personnel regarding COVID 19.

STUDY POPULATION: Nursing Personnel who were aged 18 years and above were included in the study.

STUDY PROCEDURE: Due to the country's lockdown at the time of data collection, WhatsApp and Facebook Messenger were used to collect the data. A total of 216 nursing personnel who were members in the several WhatsApp and Facebook Messenger groups were approached to participate in the study. An online data collection tool was developed and accomplished using Google Forms (via docs.google.com/forms). The Google Form link to the questionnaire was sent to the enrolled participants via the identified WhatsApp and Facebook Messenger groups.

\section{TOOL FOR DATA COLLECTION:}

Data collection tool contain items on the following aspects:

PART 1: Socio Demographic Profile

PART 2: Structured knowledge questionnaire regarding COVID 19.

PART 3: Practice Checklist Questionnaire regarding COVID 19.

\section{Tool 1: Socio demographic Profile}

The purpose of the tool was to identify the profile of the nursing personnel. It contains the question of socio demographic variables comprising of age, gender, education, occupation, experience, area of living, source of information regarding COVID 19 and COVID 19 exposure in social circle.

Tool 2: Structured knowledge questionnaire on COVID 19:

The structured knowledge questionnaire was developed to determine the knowledge of nursing personnel on COVID 19. This questionnaire has 30 multiple choice questions on COVID 19. Each question has four choices: one right choice, three wrong choices. The correct choice carries one point and other choices will be scored as ' 0 '. The range of score for an individual will be between 0 and 30:-

- 0-6

- 7-12

- 13-18

- 19-24

- 25-30

\section{Very Poor : Poor : $\quad$ Average : $\quad$ Good : $\quad$ Very Good}

Tool 3: Structured Practice Checklist:

It will consist of 15 items related to practices viz. preventive measure of COVID 19. The total score will range from 0 to 15 .

\begin{tabular}{llll}
- & $0-5$ & $:$ & \multicolumn{2}{c}{ Need Practice } \\
- & $6-10$ & : & Satisfactory \\
- & $11-15$ & : & Excellent
\end{tabular}




\section{RESULT}

Total 216 nursing personnel approached, 200 participants responded to the questionnaire. Mostly (90.5\%) of the samples belong to age group of 18-27 years. $90.5 \%$ of the samples were female and $41.5 \%$ samples live in urban area. Majority $(59 \%)$ of the samples were graduate.
In occupation distribution $58.5 \%$ of nursing personnel were student whereas $24 \%$ were working in clinical field and only $17.5 \%$ were working in teaching field. Majority $95.5 \%$ of samples had information regarding COVID-19. Among those samples $51.4 \%$ got information through social media. Only $21.5 \%$ of nursing personnel got exposure to COVID-19.

\begin{tabular}{|c|c|c|c|}
\hline S. No. & Demographic Variables & $\begin{array}{l}\text { Frequency } \\
\text { (f) }\end{array}$ & $\begin{array}{c}\text { Percentage } \\
(\%)\end{array}$ \\
\hline 1 & $\begin{array}{l}\text { Age in years } \\
\text { a) } 18-27 \\
\text { b) } 28-37 \\
\text { c) } 38-47 \\
\text { d) Above } 47\end{array}$ & $\begin{array}{c}181 \\
18 \\
1 \\
0\end{array}$ & $\begin{array}{c}90.5 \\
9 \\
0.5 \\
0\end{array}$ \\
\hline 2 & $\begin{array}{cc} & \text { Gender } \\
\text { a) } & \text { Male } \\
\text { b) } & \text { Female } \\
\text { c) } & \text { Transgender }\end{array}$ & $\begin{array}{c}19 \\
181 \\
0\end{array}$ & $\begin{array}{c}9.5 \\
90.5 \\
0\end{array}$ \\
\hline 3 & 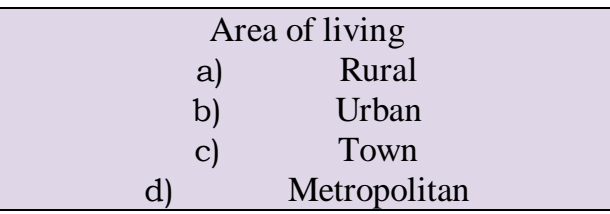 & $\begin{array}{l}67 \\
83 \\
26 \\
24\end{array}$ & $\begin{array}{c}33.5 \\
41.5 \\
13 \\
12 \\
\end{array}$ \\
\hline 4 & $\begin{array}{cc} & \text { Education } \\
\text { a) } & \text { Diploma } \\
\text { b) } & \text { Graduate } \\
\text { c) } & \text { Post graduate } \\
\text { d) } & \text { Doctorate } \\
\end{array}$ & $\begin{array}{c}55 \\
118 \\
27 \\
0\end{array}$ & $\begin{array}{c}27.5 \\
59 \\
13.5 \\
0\end{array}$ \\
\hline 5 & $\begin{array}{cc} & \text { Occupation } \\
\text { a) } & \text { Teaching } \\
\text { b) } & \text { Clinical } \\
\text { c) } & \text { Student }\end{array}$ & $\begin{array}{l}35 \\
48 \\
117\end{array}$ & $\begin{array}{c}17.5 \\
24 \\
58.5\end{array}$ \\
\hline 6 & 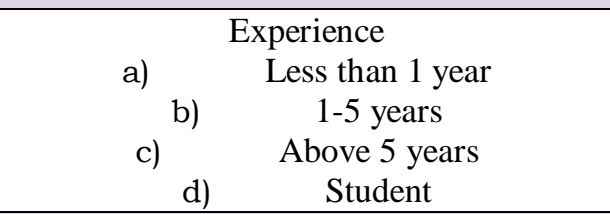 & $\begin{array}{c}37 \\
43 \\
12 \\
108\end{array}$ & $\begin{array}{c}18.5 \\
21.5 \\
6 \\
54\end{array}$ \\
\hline 7. A & $\begin{array}{l}\text { Do you have any information regarding } \\
\text { COVID-19? } \\
\begin{array}{ll}\text { a) } & \text { Yes } \\
\text { b) } & \text { No }\end{array}\end{array}$ & $\begin{array}{c}191 \\
9\end{array}$ & $\begin{array}{c}95.5 \\
4.5\end{array}$ \\
\hline 7. $\mathrm{B}$ & $\begin{array}{l}\text { If yes, the source of information regarding } \\
\text { COVID-19 is:- } \\
\begin{aligned} \text { a) } & \text { Social Media } \\
\text { b) } & \text { Peer group } \\
\text { c) } & \text { Conference/workshop } \\
\text { d) } & \text { TV/ Newspaper } \\
\text { e) } & \text { Online Training } \\
\text { f) } & \text { Any other (Specify) }\end{aligned}\end{array}$ & $\begin{array}{c}98 \\
5 \\
5 \\
69 \\
11 \\
3 \\
\end{array}$ & $\begin{array}{c}51.4 \\
2.6 \\
2.6 \\
36.2 \\
5.7 \\
1.5 \\
\end{array}$ \\
\hline 8. A & 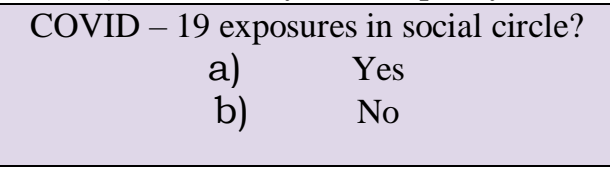 & $\begin{array}{c}43 \\
157\end{array}$ & $\begin{array}{l}21.5 \\
78.5\end{array}$ \\
\hline
\end{tabular}




\begin{tabular}{|c|c|c|c|}
\hline 8. B & 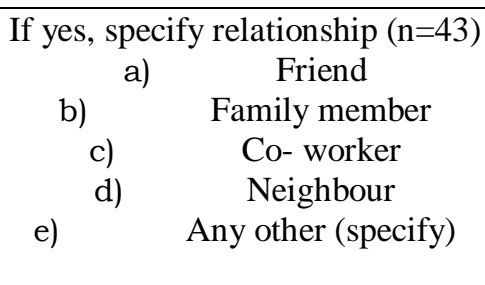 & $\begin{array}{c}11 \\
9 \\
11 \\
9 \\
3\end{array}$ & $\begin{array}{c}25.6 \\
20.9 \\
25.6 \\
20.9 \\
7\end{array}$ \\
\hline
\end{tabular}

Table 1:- Frequency and Percentage Distribution of samples based on Demographic Variables.

$$
\mathrm{N}=200
$$

\begin{tabular}{|c|c|c|c|}
\hline Grading of Scores & Grading of Knowledge & Frequency (f) & Percentage (\%) \\
\hline $\mathbf{0 - 6}$ & Very Poor & 1 & 0.5 \\
\hline $\mathbf{7 - 1 2}$ & Poor & 89 & 9 \\
\hline $\mathbf{1 3 - 1 8}$ & Average & 87 & 44.5 \\
\hline $\mathbf{1 9 - 2 4}$ & Good & 5 & 43.5 \\
\hline $\mathbf{2 5 - 3 0}$ & Very Good & 2.5 \\
\hline
\end{tabular}

Table 2:- Frequency and percentage distribution of samples based on level of knowledge.

$$
\mathrm{N}=200
$$

Table no. 2 depicts that $44.5 \%$ of samples had average knowledge whereas $43.5 \%$ had good knowledge and $9 \%$ of the samples had poor knowledge. Only $0.5 \%$ samples had very poor knowledge.

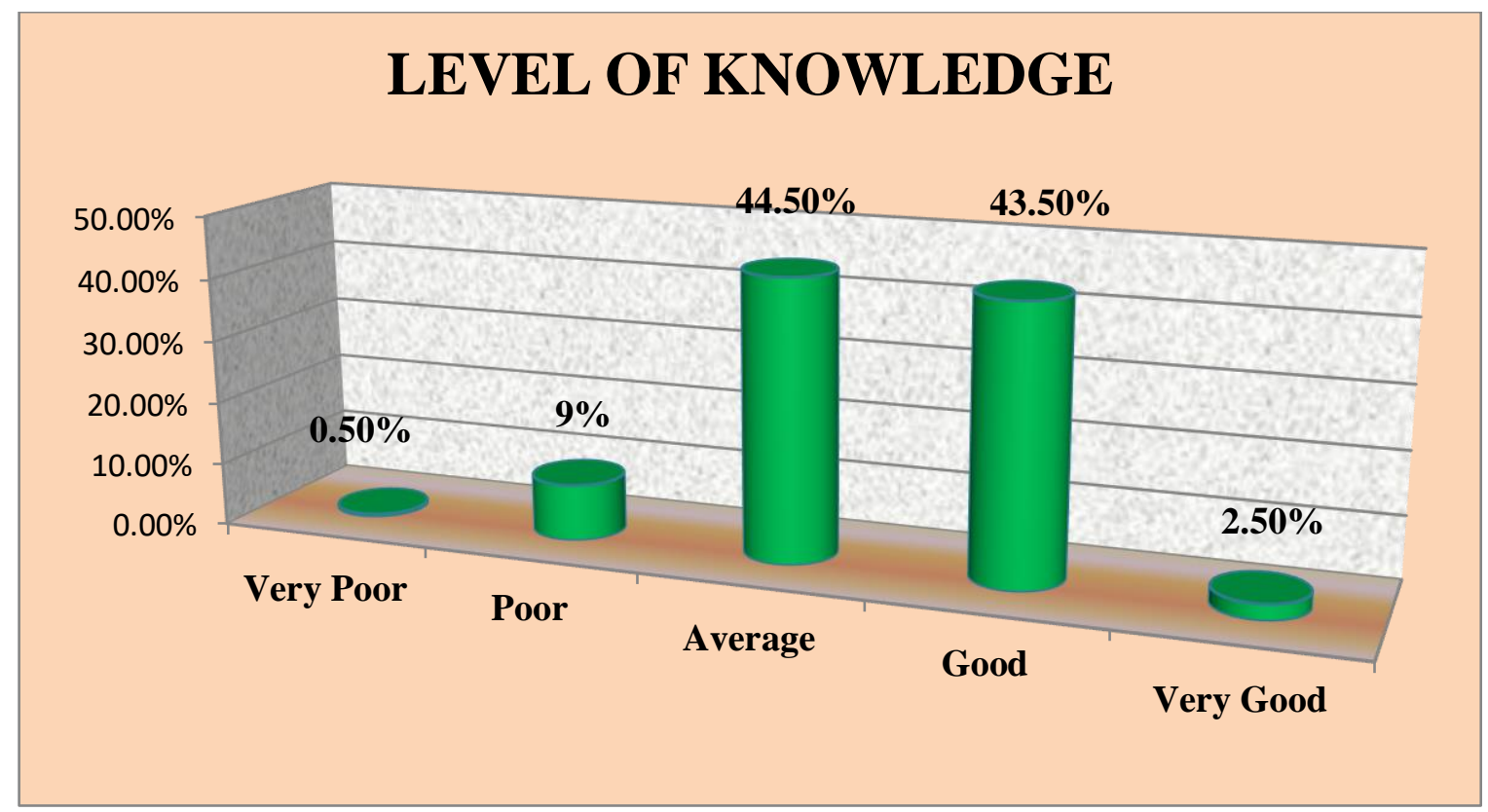

Fig 1:- Diagrammatic Presentation of samples based on level of knowledge.

\begin{tabular}{|c|c|c|c|}
\hline \multirow{2}{*}{$\begin{array}{c}\text { Grading of Scores } \\
\text { (-5 }\end{array}$} & Need Practice & 0 & 0 \\
\hline $\mathbf{6 - 1 0}$ & Satisfactory & 7 & 3.5 \\
\hline $\mathbf{1 1 - 1 5}$ & Excellent & 193 & 96.5 \\
\hline
\end{tabular}

Table 3:- Frequency and percentage distribution of samples based on levels of practice.

$$
\mathrm{N}=200
$$

Table no. 3 depicts that majority $(96.5 \%)$ of samples had Excellent practice whereas only $3.5 \%$ samples had satisfactory practice. 


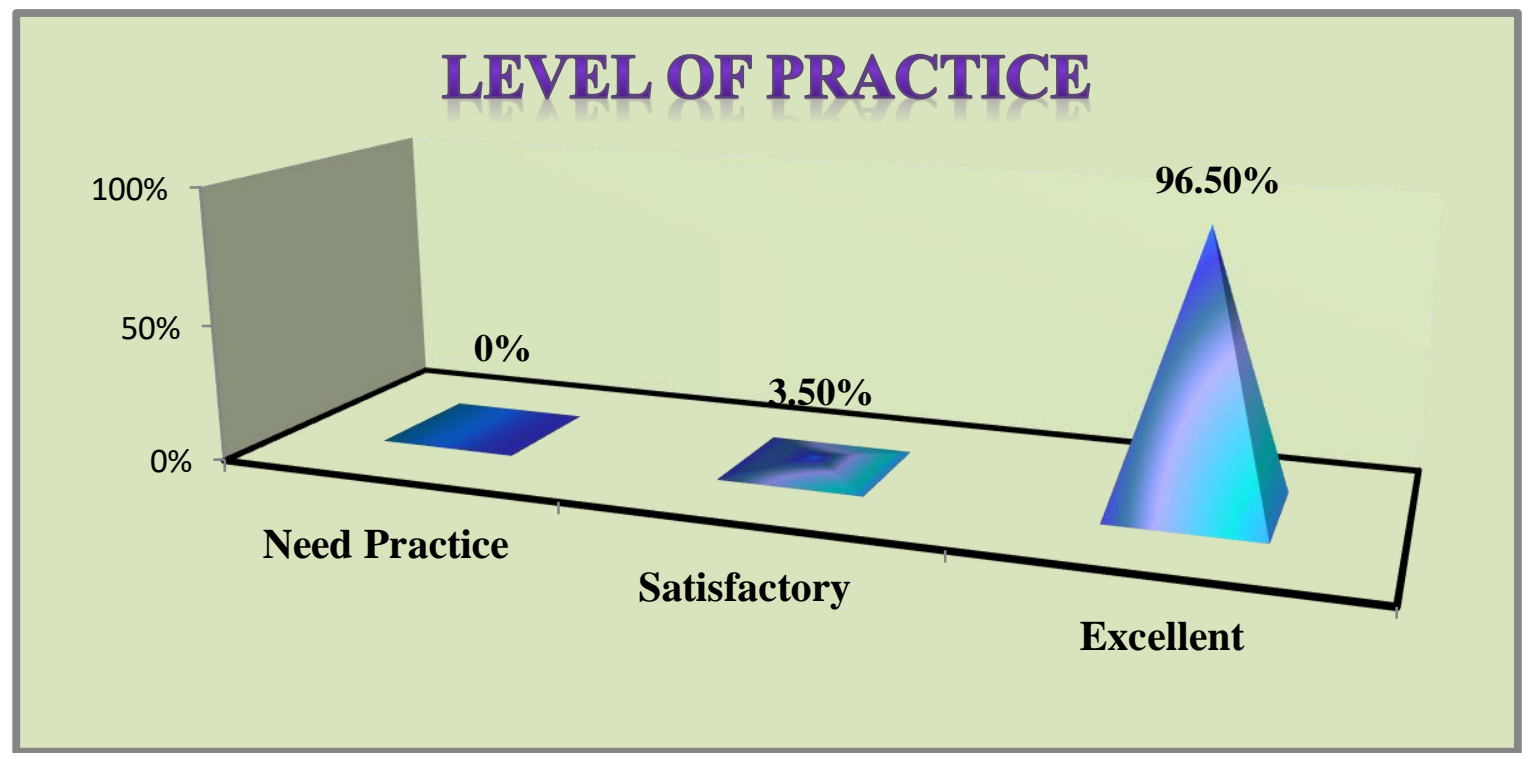

Fig 2:- Diagrammatic presentation of samples based on level of practice.

\begin{tabular}{|c|c|c|c|c|}
\hline S. No. & $\begin{array}{l}\text { Mean } \\
\text { score }\end{array}$ & $\begin{array}{l}\text { Degree of } \\
\text { freedom }\end{array}$ & 'r' value & P Value \\
\hline Mean knowledge score & 17.78 & & & $\begin{array}{l}\text { The } \mathrm{P} \text {-Value is } 0.000053 \text {. The } \\
\text { result is significant at } \mathrm{p}\end{array}$ \\
\hline Mean Practice Score & 13.89 & 199 & 0.2817 & Weak positive correlation. \\
\hline
\end{tabular}

Note: $\mathbf{r}>\mathbf{0}$ (weak positive correlation)

Table 4:- Correlation between the knowledge and practice.

$$
\mathrm{N}=200
$$

The above table depicts that the obtained $r$ value 0.28 which is greater than 0 . Therefore $r$ value indicate weak positive correlation between the knowledge and practice score thus the research hypothesis stated that there is a significant positive correlation between knowledge score and practice score of nursing personnel regarding COVID -19 was accepted.

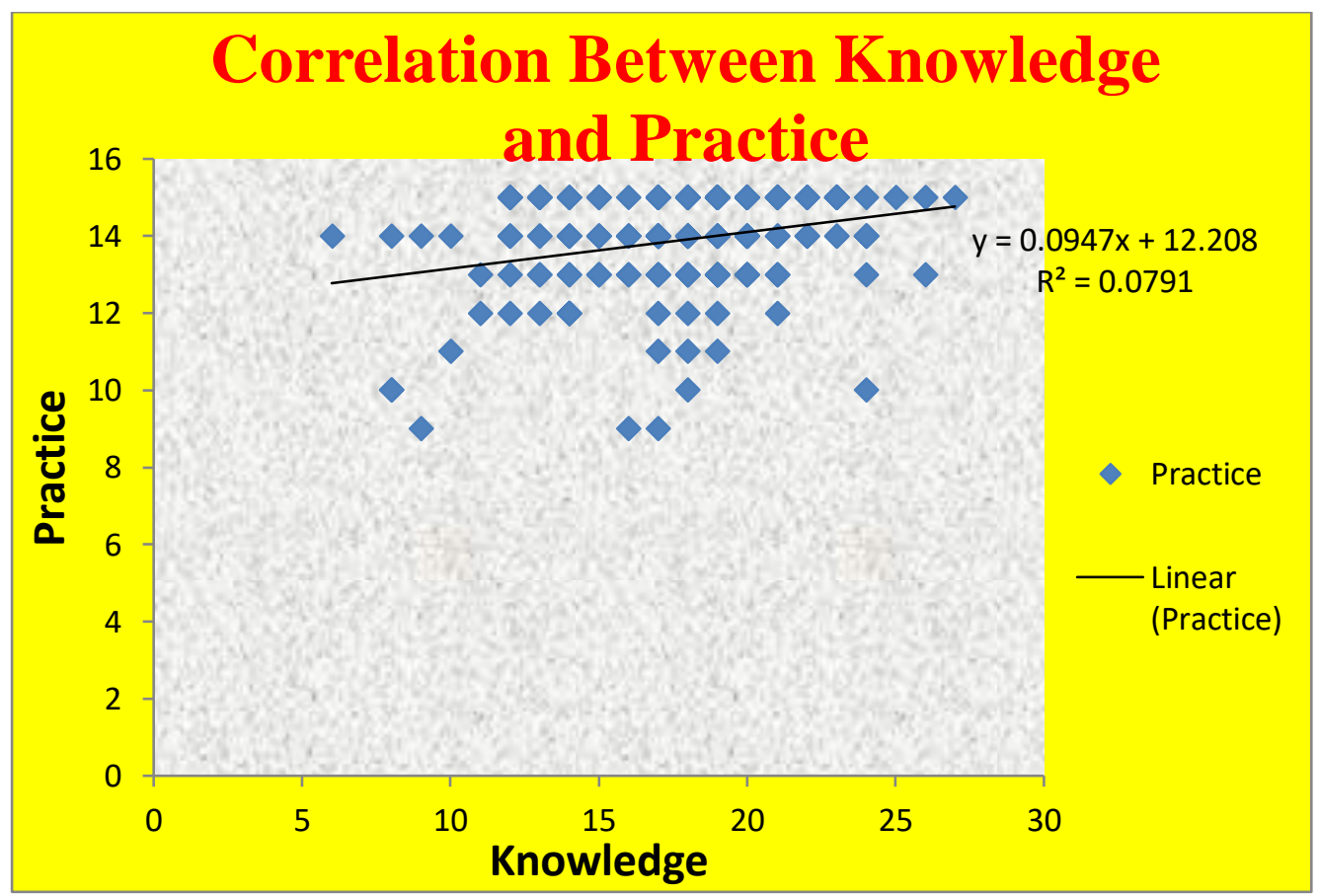

Fig 3:- Diagrammatic presentation of correlation between knowledge and practice. 
ISSN No:-2456-2165

\begin{tabular}{|c|c|c|c|c|c|c|}
\hline \multirow{2}{*}{ S. No. } & \multirow{2}{*}{ Demographic Variables } & \multicolumn{2}{|c|}{ Knowledge Score } & \multirow{2}{*}{$\begin{array}{c}\text { CHI } \\
\text { Square Value }\end{array}$} & \multirow{2}{*}{ P Value } & \multirow{2}{*}{$\mathbf{S} / \mathbf{N S}$} \\
\hline & & $<$ Mean & $\geq$ Mean & & & \\
\hline 1 & $\begin{array}{l}\text { Age in years } \\
\text { a) } 18-27 \\
\text { b) } 28-37 \\
\text { c) } 38-47 \\
\text { d) Above } 47\end{array}$ & $\begin{array}{c}81 \\
6 \\
1 \\
0\end{array}$ & $\begin{array}{c}100 \\
12 \\
0 \\
0\end{array}$ & 2.14 & 0.5428 & NS \\
\hline 2 & $\begin{array}{cc} & \text { Gender } \\
\text { a) } & \text { Male } \\
\text { b) } & \text { Female } \\
\text { c) } & \text { Transgender }\end{array}$ & $\begin{array}{c}6 \\
82 \\
0\end{array}$ & $\begin{array}{c}13 \\
99 \\
0\end{array}$ & 1.31 & 0.2515 & NS \\
\hline 3 & \begin{tabular}{cc} 
& \multicolumn{2}{c}{ Area of living } \\
a) & Rural \\
b) & Urban \\
c) & Town \\
d) & Metropolitan
\end{tabular} & $\begin{array}{c}34 \\
33 \\
13 \\
8\end{array}$ & $\begin{array}{l}33 \\
50 \\
13 \\
16\end{array}$ & 3.33 & 0.3432 & NS \\
\hline 4 & $\begin{array}{cc} & \text { Education } \\
\text { a) } & \text { Diploma } \\
\text { b) } & \text { Graduate } \\
\text { c) } & \text { Post graduate } \\
\text { d) } & \text { Doctorate }\end{array}$ & $\begin{array}{c}44 \\
38 \\
6 \\
0\end{array}$ & $\begin{array}{c}11 \\
80 \\
21 \\
0\end{array}$ & 40.79 & 0.00001 & $S$ \\
\hline 5 & \begin{tabular}{cr}
\multicolumn{2}{c}{ Occupation } \\
a) & Teaching \\
b) & Clinical \\
c) & Student
\end{tabular} & $\begin{array}{l}10 \\
19 \\
59\end{array}$ & $\begin{array}{l}25 \\
29 \\
58\end{array}$ & 5.72 & 0.0571 & NS \\
\hline 6 & 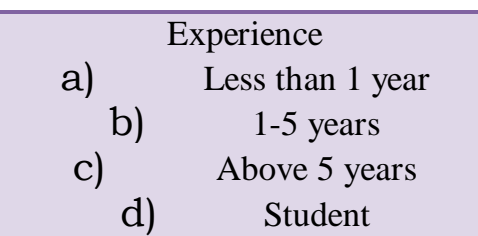 & $\begin{array}{c}17 \\
15 \\
2 \\
54\end{array}$ & $\begin{array}{l}20 \\
28 \\
10 \\
54\end{array}$ & 6.72 & 0.0812 & NS \\
\hline 7. $\mathrm{A}$ & $\begin{array}{l}\text { Do you have any information } \\
\text { regarding COVID-19? } \\
\begin{array}{ll}\text { a) } & \text { Yes } \\
\text { b) } & \text { No }\end{array}\end{array}$ & $\begin{array}{c}80 \\
8\end{array}$ & $\begin{array}{c}111 \\
1\end{array}$ & 7.70 & 0.0055 & S \\
\hline 7. $\mathrm{B}$ & 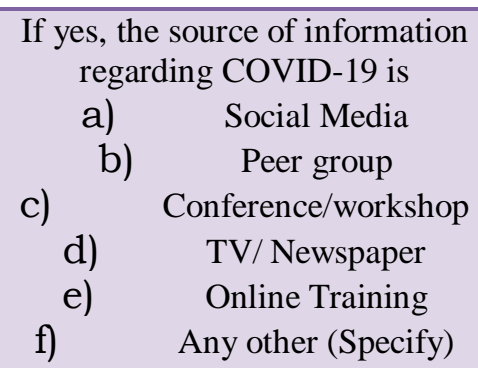 & $\begin{array}{c}41 \\
3 \\
2 \\
32 \\
2 \\
0\end{array}$ & $\begin{array}{c}57 \\
2 \\
3 \\
37 \\
9 \\
3\end{array}$ & 5.95 & 0.3106 & NS \\
\hline 8. A & $\begin{array}{l}\text { COVID - } 19 \text { exposures in social } \\
\text { circle? } \\
\begin{array}{cc}\text { a) } & \text { Yes } \\
\text { b) } & \text { No }\end{array}\end{array}$ & $\begin{array}{l}31 \\
57\end{array}$ & $\begin{array}{c}12 \\
100\end{array}$ & 17.54 & 0.00002 & S \\
\hline
\end{tabular}




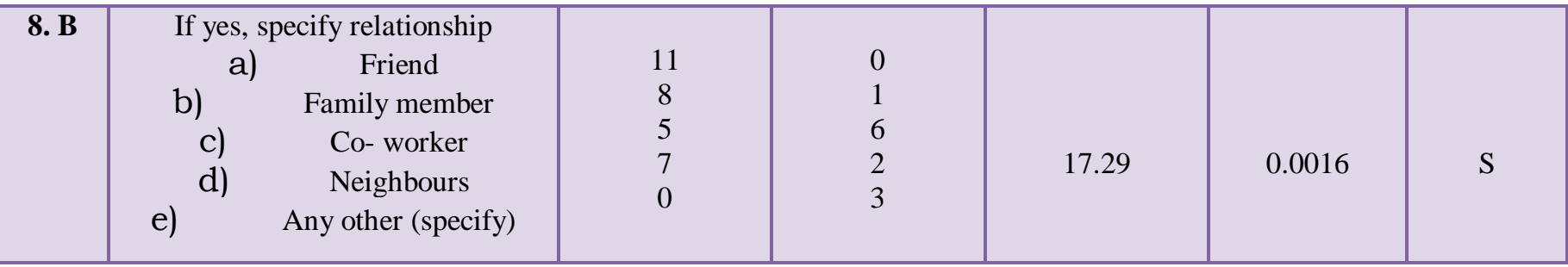

Note: $\mathbf{p} \leq \mathbf{0 . 0 5}, \mathrm{S}$ - Significant, $\mathrm{NS}$ - Non significant

Table 5:- Association of knowledge score of samples with their selected Demographic variables.

$$
\mathrm{N}=200
$$

The data is presented in table 5 showed that there was significant association between Knowledge and Education $\left(\chi^{2}(3,0.00001)\right.$ $=40.79)$, Information on COVID $-19\left(\chi_{(1,0.005)}^{2}=7.70\right)$, Exposure to COVID-19 $\left(\chi_{(1,0.00002)}^{2}=17.34\right)$ and Relationship of exposure $\left(\chi_{(5,0.0016)}^{2}=17.29\right)$. Thus it can be interpreted there was significant association between knowledge on COVID-19 and selected demographic variable in relation to education, information on COVID -19, Exposure to COVID-19 and relationship with exposure. Therefore the research hypothesis is accepted. There was no significant association between knowledge and other variable. Thus the research hypothesis is rejected.

\begin{tabular}{|c|c|c|c|c|c|c|}
\hline \multirow{2}{*}{ S. No. } & \multirow{2}{*}{ Demographic Variables } & \multicolumn{2}{|c|}{ Knowledge Score } & \multirow{2}{*}{$\begin{array}{c}\text { CHI } \\
\text { Square } \\
\text { Value }\end{array}$} & \multirow{2}{*}{ P Value } & \multirow{2}{*}{$\mathbf{S} / \mathbf{N S}$} \\
\hline & & $<$ Mean & $\geq$ Mean & & & \\
\hline 1 & $\begin{array}{l}\text { Age in years } \\
\text { a) } 18-27 \\
\text { b) } 28-37 \\
\text { c) } 38-47 \\
\text { d) Above } 47\end{array}$ & $\begin{array}{c}49 \\
5 \\
1 \\
0\end{array}$ & $\begin{array}{c}132 \\
13 \\
0 \\
0\end{array}$ & 2.65 & 0.4481 & NS \\
\hline 2 & $\begin{array}{cc} & \text { Gender } \\
\text { a) } & \text { Male } \\
\text { b) } & \text { Female } \\
\text { c) } & \text { Transgender }\end{array}$ & $\begin{array}{c}5 \\
50 \\
0\end{array}$ & $\begin{array}{c}14 \\
131 \\
0\end{array}$ & 0.01 & 0.9925 & NS \\
\hline 3 & \begin{tabular}{cc}
\multicolumn{2}{c}{ Area of living } \\
a) & Rural \\
b) & Urban \\
c) & Town \\
d) & Metropolitan
\end{tabular} & $\begin{array}{l}18 \\
24 \\
10 \\
3\end{array}$ & $\begin{array}{l}49 \\
59 \\
16 \\
21\end{array}$ & 4.37 & 0.2239 & NS \\
\hline 4 & $\begin{array}{cc} & \text { Education } \\
\text { a) } & \text { Diploma } \\
\text { b) } & \text { Graduate } \\
\text { c) } & \text { Post graduate } \\
\text { d) } & \text { Doctorate } \\
\end{array}$ & $\begin{array}{c}19 \\
28 \\
8 \\
0\end{array}$ & $\begin{array}{c}36 \\
90 \\
19 \\
0\end{array}$ & 2.27 & 0.517 & NS \\
\hline 5 & \begin{tabular}{cc}
\multicolumn{2}{c}{ Occupation } \\
a) & Teaching \\
b) & Clinical \\
c) & Student \\
\end{tabular} & $\begin{array}{c}6 \\
16 \\
33\end{array}$ & $\begin{array}{l}29 \\
32 \\
84\end{array}$ & 2.73 & 0.2551 & NS \\
\hline 6 & 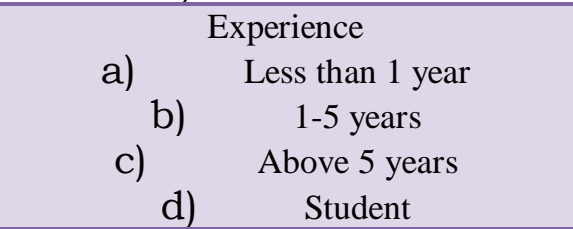 & $\begin{array}{c}12 \\
11 \\
3 \\
29\end{array}$ & $\begin{array}{c}25 \\
32 \\
9 \\
79\end{array}$ & 0.59 & 0.8984 & NS \\
\hline 7. A & $\begin{array}{l}\text { Do you have any information regarding } \\
\text { COVID-19? } \\
\begin{array}{ll}\text { a) } & \text { Yes } \\
\text { b) } & \text { No }\end{array}\end{array}$ & $\begin{array}{c}54 \\
1\end{array}$ & $\begin{array}{c}137 \\
8\end{array}$ & 1.27 & 0.2597 & NS \\
\hline
\end{tabular}




\begin{tabular}{|c|c|c|c|c|c|c|}
\hline 7. $B$ & $\begin{array}{l}\text { If yes, the source of information regarding } \\
\text { COVID-19 is } \\
\begin{aligned} \text { a) } & \text { Social Media } \\
\text { b) } & \text { Peer group } \\
\text { c) } & \text { Conference/workshop } \\
\text { d) } & \text { TV/ Newspaper } \\
\text { e) } & \text { Online Training } \\
\text { f) } & \text { Any other (Specify) }\end{aligned}\end{array}$ & $\begin{array}{c}31 \\
3 \\
2 \\
17 \\
0 \\
1\end{array}$ & $\begin{array}{c}67 \\
2 \\
3 \\
52 \\
11 \\
2\end{array}$ & 8.19 & 0.1460 & NS \\
\hline 8. A & 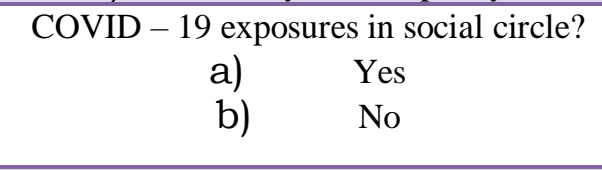 & $\begin{array}{l}17 \\
38\end{array}$ & $\begin{array}{c}26 \\
119\end{array}$ & 3.97 & 0.0460 & $\mathrm{~S}$ \\
\hline 8. B & 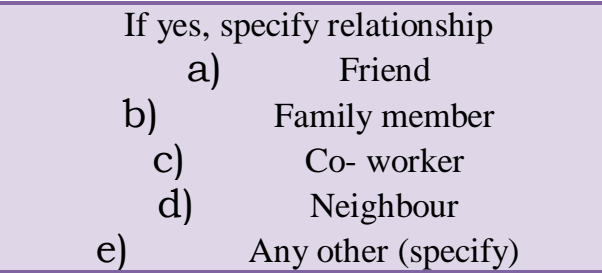 & $\begin{array}{l}6 \\
3 \\
5 \\
1 \\
2\end{array}$ & $\begin{array}{l}5 \\
6 \\
6 \\
8 \\
1\end{array}$ & 5.30 & 0.2571 & NS \\
\hline
\end{tabular}

Note: $\mathbf{p} \leq \mathbf{0 . 0 5}, \mathrm{S}$ - Significant, NS - Non significant

Table 6:- Association of Practice Score of samples with their selected Demographic variables

$$
\mathrm{N}=200
$$

The data presented in table 6 showed that there was significant association between Practice and Exposure to COVID-19 $\left(\chi_{(1,0.0460)}^{2}=3.97\right)$. Thus it can be interpreted there was significant association between Practice on COVID-19 and selected demographic variable in relation to Exposure to COVID-19. Therefore the research hypothesis is accepted. There was no significant association between Practice and other variable. Thus the research hypothesis is rejected.

\section{CONCLUSION}

The study concluded that continuing nursing education is advised to improve the knowledge of nursing professional hence preventive and therapeutic practices can be encouraged.

\section{REFERENCES}

[1]. Coronavirus COVID-19 Global Cases". Johns Hopkins University. Retrieved 20 March 2020.

[2]. Coronavirus Update (Live): 307,627 Cases and 13,050 Deaths from COVID-19 Virus OutbreakWorldometer". www.worldometers.info.

[3]. Coronavirus disease 2019. World Health Organization. Retrieved 15 March 2020.

[4]. Coronavirus Disease 2019 (COVID-19) Transmission". Centers for Disease Control and Prevention. 17 March 2020. Retrieved 23 March 2020.

[5]. CDC. CDC Confirms Possible Instance of Community Spread of COVID-19 in U.S. Novel Coronavirus (2019-nCoV), Wuhan, China. Coronavirus Disease 2019 (COVID-19).

[6]. Protocol for assessment of potential risk factors for 2019-novel coronavirus (2019-nCoV) infection among health care workers in a health care setting. Available from: https://www.who.int/publicationsdetail/infectionprevention-and-control-during-health-care-when-novelcoronavirus-(ncov)-infection-is-suspected

[7]. Mohammed Ikmal, Sudarshan Paudel, Ya-Wen Chiu, Chinenye Nwoke et. al. Knowledge and Perceptions of COVID-19 Among Health Care Workers: CrossSectional Study. JMIR Public Health Surveill. 2020 Apr-Jun; 6(2): e19160. PMCID: PMC7193987.

[8]. COVID-19: protecting health-care workers. The lancet. DOI-6736(20)30627. PIIS0140-6736(20) 30627-9. Vol 395, March 21, 2020. Available from: https://www.thelancet.com /journals/lancet/ article.

[9]. Erika Edwards. COVID-19 cases among health care workers top 62,000, CDC reports. Availablefrom:https://www.nbcnews.com/health/healt h-news/covid-19-cases-among-health-care-workerstop62 000-n1215056.

[10]. Ronald Olum, Zisis Kozlakidis et. al. Coronavirus Disease-2019: Knowledge, Attitude, and Practices of Health Care Workers at Makerere University Teaching Hospitals, Uganda. Front. Public Health 8:181. doi: 10.3389/fpubh.2020.00181.

[11]. Polit F. Denise and Beck Cheryl Tatano. Nursing Research, generating and assessing evidence for nursing practice. Tenth edition. New Delhi: Wolters Kluwer India Pvt Ltd; 2016. 